\title{
Rotational properties of non-dipolar and dipolar Bose-Einstein condensates confined in annular potentials
}

\author{
E. Ö. Karabulut ${ }^{1,4}$, F. Malet ${ }^{2}$, G. M. Kavoulakis ${ }^{3}$, and S. M. Reimann ${ }^{1}$ \\ ${ }^{1}$ Mathematical Physics, Lund University, LTH, P.O. Box 118, SE-22100 Lund, Sweden \\ ${ }^{2}$ Department of Theoretical Chemistry and Amsterdam Center for Multiscale Modeling, \\ FEW, Vrije Universiteit, De Boelelaan 1083, 1081HV Amsterdam, The Netherlands \\ ${ }^{3}$ Technological Educational Institute of Crete, P.O. Box 1939, GR-71004, Heraklion, Greece \\ ${ }^{4}$ Physics Department, Faculty of Science, Selcuk University, TR-42075, Konya, Turkey
}

(Dated: July 4, 2018)

\begin{abstract}
We investigate the rotational response of both non-dipolar and dipolar Bose-Einstein condensates confined in an annular potential. For the non-dipolar case we identify certain critical rotational frequencies associated with the formation of vortices. For the dipolar case, assuming that the dipoles are aligned along some arbitrary and tunable direction, we study the same problem as a function of the orientation angle of the dipole moment of the atoms.

PACS numbers: 03.75.Lm, 05.30.Jp
\end{abstract}

\section{INTRODUCTION}

In recent experiments on ultra-cold atomic gases the confinement of atoms in toroidal traps has become possible [1]. In such confining potentials persistent currents have also been created and observed [2 5], in close analogy to semiconductor nanostructures [6, 7]. The simplicity of these systems makes them ideal for studying effects associated with superfluidity, including rotational properties, persistent flow, etc.

In another series of recent experiments it has become possible to trap atoms [8] and molecules [9] with a nonzero dipole moment. Contrary to most of the previously realized experiments, in which the particles had zero dipole moment and the usual contact interaction modeled the effective atom-atom/molecule-molecule interaction rather well, in the dipolar case the interaction potential is long-ranged and anisotropic. As a consequence of these differences, dipolar gases present new properties that allow for the study of novel and interesting effects.

In three dimensions the head-to-tail alignment of the dipoles causes instabilities towards collapse [10], while low-dimensional confinement tends to make the system more stable [11]. Quasi-two- or quasi-one-dimensional confinement thus suppresses the instabilities associated with the dipolar interaction and is advantageous in that respect.

Recently, several properties of dipolar gases have been examined in effectively two-dimensional systems. Among these, the rotational properties of dipolar degenerate gases have received considerable attention. In particular, the rotational properties of dipolar Bose-Einstein condensates have been investigated in different confining geometries, including axially-symmetric harmonic [12], elliptic harmonic [13], and toroidal traps [14 18]. Also, recently it has become possible to create a Bose-Einstein condensate of dysprosium 164, with a magnetic moment of roughly three times larger than chromium 52, which increases the dipole coupling constant by roughly a factor of ten [19].

Dipolar Bose-Einstein condensates confined in toroidal traps have been investigated both within the mean-field approximation [14], and in the few-particle limit [20]. These systems have been shown to exhibit some very interesting effects due to the interplay between the nontrivial topology of the ring-like confinement and the anisotropic nature of the dipole-dipole interaction. More specifically, when the dipole moments have a non-zero in-plane component, the effects associated with breaking the axial symmetry of the system have been shown to have potential applications in, e.g., Josephson-type oscillations and self-trapping phenomena [16, 17, 21].

Motivated by the experimental progress mentioned above on toroidal traps and on dipolar gases, we study below the rotational properties of a Bose-Einstein condensate that is confined in an annular-like potential, first considering a condensate that consists of non-dipolar atoms [22-24] and then addressing the dipolar case. Our calculations are closely-related with those of Ref. [25], which analysed the lowest-energy state of a uniform (nondipolar) rotating superfluid confined in a hard-wall annulus. In this study it was shown that as the rotational frequency increases initially there are vortices that are located in the region of zero density. As the rotational frequency increases, vortices start to form in the region of nonzero density. With increasing angular frequency of the trap the real vortices eventually form a circular array, or even multiple arrays of vortices. These theoretical predictions were in good agreement with the experimental results on liquid Helium that followed afterwards [26]. In what follows below we refer to the vortices that are located in the region of negligible density as "phantom" vortices and the ones that are located in the region of non-negligible density as "real" vortices.

The paper is organized as follows. In Sec. II. we present our model. In Sec. III we investigate the rotational response of a non-dipolar gas. In the spirit of Ref. [25] we identify the corresponding critical rotational frequencies. In Sec. IV we investigate the effect of the dipolar inter- 


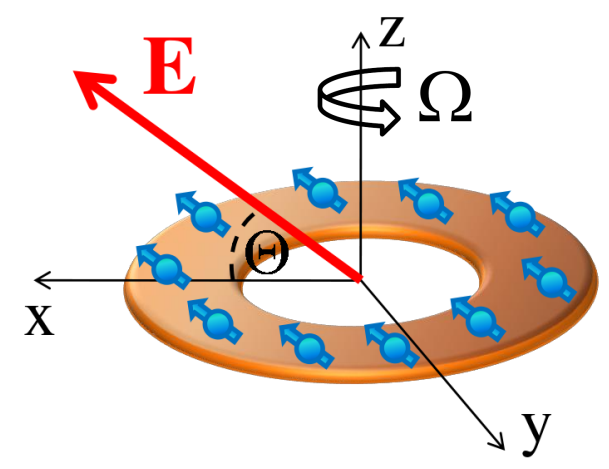

FIG. 1: (Color online) Schematic illustration of the rotating quasi-two-dimensional annular trap. The atoms move on the $x-y$ plane with an angular frequency $\Omega$, which is along the $z$ axis, in an annulus of mean radius $R$ and a width that is set by the oscillator length $a_{0}=\sqrt{\hbar /\left(M \omega_{0}\right)}$. In the case of dipolar atoms, their dipole moment is oriented on the $x-z$ plane, forming an angle $\Theta$ with the $x$ axis due to an external (magnetic or electric) field $\mathbf{E}$.

action, assuming that the dipole moment of the atoms is oriented along some arbitrary direction due to an external polarizing field. We demonstrate that the vortex structures depend strongly on the orientation angle of the dipole moment of the atoms. Finally in Sec. V we summarize our results.

\section{MODEL}

The annular potential that we consider is modeled via a combination of harmonic potentials in the transverse direction and in the direction perpendicular to the plane of motion of the atoms,

$V(\mathbf{r})=V_{r}\left(\mathbf{r}_{\perp}\right)+V_{z}(z)=\frac{1}{2} M \omega_{0}^{2}\left(r_{\perp}-R\right)^{2}+\frac{1}{2} M \omega_{z}^{2} z^{2}$.

Here $z$ is the symmetry axis of the trap, which is also the axis of rotation of the annulus. In addition $\mathbf{r}_{\perp}$ is the position vector on the $x-y$ plane, while $\omega_{0}$ and $\omega_{z}$ are the trap frequencies. The ratio $\omega_{z} / \omega_{0}$ is chosen to be equal to 100 , which makes the motion of atoms quasi-two-dimensional, since $\hbar \omega_{z}$ is the largest energy scale in the problem. The ratio $R / a_{0}$ is chosen equal to 4 , where $a_{0}=\sqrt{\hbar /\left(M \omega_{0}\right)}$ is the oscillator length that corresponds to the frequency $\omega_{0}$ and mass $M$. Figure 1 shows schematically the corresponding annular-like potential and also the external field that is assumed to polarize the (dipolar) atoms. Without loss of generality, this field is taken to be on the $x z$-plane, forming an angle $\Theta$ with the $x$-axis.

Concerning the interactions, these are modeled via a short-ranged contact potential - which takes care of the short-range correlations - and the usual dipole-dipole potential, which describes the long-range part of the interaction. Because of the assumed strong confinement along the $z$-axis, one may safely assume that the order parameter has a product form

$$
\Psi(\mathbf{r})=\Phi\left(\mathbf{r}_{\perp}\right) \times \phi(z),
$$

where $\phi(z)$ is the Gaussian ground state of the harmonic potential $V_{z}(z)$. This assumption allows us to reduce the problem from three dimensions to two dimensions for the order parameter $\Phi\left(\mathbf{r}_{\perp}\right)$, which satisfies the GrossPitaevskii-like equation

$$
\begin{aligned}
{\left[-\frac{\hbar^{2} \nabla_{\perp}^{2}}{2 M}+V_{r}\left(\mathbf{r}_{\perp}\right)+V_{\operatorname{dip}}\left(\mathbf{r}_{\perp}\right)+g\left|\Phi\left(\mathbf{r}_{\perp}\right)\right|^{2}\right] } & \Phi\left(\mathbf{r}_{\perp}\right)= \\
& =\mu \Phi\left(\mathbf{r}_{\perp}\right) .
\end{aligned}
$$

In the above equation $g=\left(\hbar^{2} / M\right) \sqrt{8 \pi} N a / a_{z}$, where $N$ is the atom number, $a$ is the scattering length for zeroenergy elastic atom-atom collisions, $a_{z}$ is the oscillator length in the $z$ direction, $\mu$ is the chemical potential, and

$$
V_{\mathrm{dip}}\left(\mathbf{r}_{\perp}\right)=\int V_{\mathrm{eff}}\left(\mathbf{r}_{\perp}-\mathbf{r}_{\perp}^{\prime}\right)\left|\Phi\left(\mathbf{r}_{\perp}^{\prime}\right)\right|^{2} d \mathbf{r}_{\perp}^{\prime}
$$

is the effective dipolar interaction potential, where $V_{\text {eff }}\left(\mathbf{r}_{\perp}-\mathbf{r}_{\perp}^{\prime}\right)$ is given in Ref. [27].

The problem thus reduces to solving the nonlocal and nonlinear integro-differential Eq. (3). We solve it with use of a fourth-order split-step Fourier method within an imaginary-time propagation approach [28]. Within this method one starts with some initial state, which then propagates in imaginary time until numerical convergence is achieved.

\section{NON-DIPOLAR ATOMS IN A ROTATING ANNULAR TRAP}

We first consider the case of non-dipolar atoms and vary the rotational frequency $\Omega$ for a fixed interaction strength, choosing $M g / \hbar^{2}=\sqrt{8 \pi} N a / a_{z}=150$. Since the ratio between the interaction energy and $\hbar \omega_{0}$ is roughly $N a a_{0} /\left(a_{z} R\right) \sim 10$, and also $\omega_{z} / \omega_{0}=100$, there is a clear hierarchy of energy scales, with $\hbar \omega_{z}$ being roughly ten times as large as the interaction energy, which in turn is roughly ten times as large as $\hbar \omega_{0}$. Therefore, while the cloud is in the ground state of the harmonic oscillator along the $z$ direction, it is closer to the Thomas-Fermi limit in the direction along the plane of motion.

Using the numerical procedure outlined above, the energetically favourable state of the cloud is determined in the rotating frame for a certain value of $\Omega$. As in Ref. 25] we identify three critical rotational frequencies $\Omega_{c 1}, \Omega_{c 2}$, and $\Omega_{c 3}$; see Fig. 2 . The first critical frequency, $\Omega_{c 1}$, corresponds to the formation of a "phantom" vortex state, which is located at the center of the annulus. These "phantom" vortices are effectively states of quantized circulation with integer quantum number. In this case the density of the cloud remains unchanged and the 
(a)

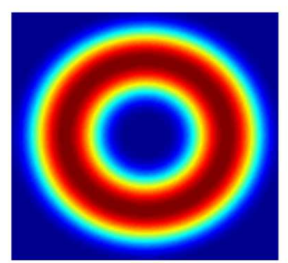

(b)
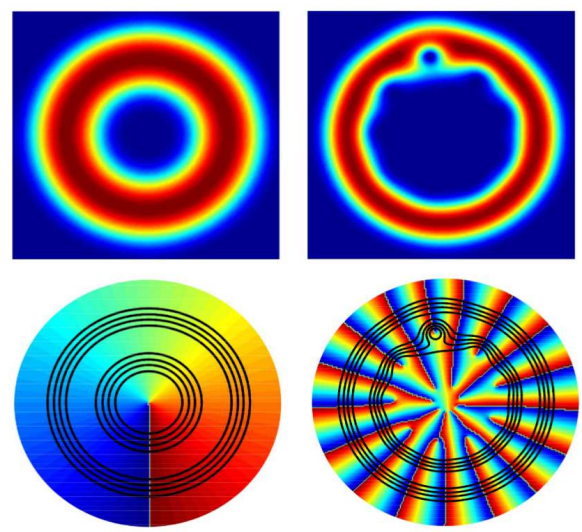

(c)
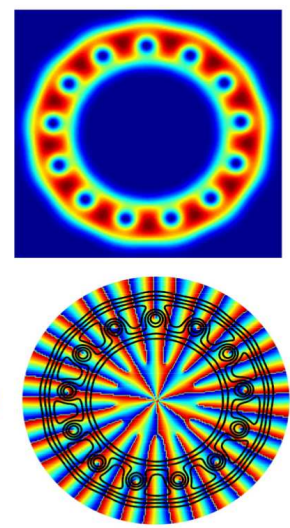

FIG. 2: (Color online) Density and phase of the order parameter corresponding to the three critical frequencies for the non-dipolar case $(D=0)$ and $\sqrt{8 \pi} N a / a_{z}=150$. For (a) $\Omega / \omega_{0}=\Omega_{c 1} / \omega_{0} \approx 0.036$ a "phantom" vortex appears, for (b) $\Omega / \omega_{0}=\Omega_{c 2} / \omega_{0} \approx 0.53$ the first "real" vortex enters the cloud, and for (c) $\Omega / \omega_{0}=\Omega_{c 3} / \omega_{0} \approx 0.63$ an array of vortices forms along the annulus.

only change takes place in the phase of the order parameter, which acquires a jump of $2 \pi$.

The second critical frequency, $\Omega_{c 2}$, is associated with the formation of the first "real" vortex. According to our results, this vortex state actually penetrates the cloud from the inner circle. The point where the vortex enters the cloud is arbitrary, as the symmetry of the Hamiltonian is broken spontaneously. Eventually more vortices penetrate the cloud and finally at the third critical frequency $\Omega_{c 3}$ an array of vortices forms. We identify $\Omega_{c 3}$ as the frequency for which the vortices are located (roughly) at the middle of the annulus. For the parameters given above $\Omega_{c 1} / \omega_{0}, \Omega_{c 2} / \omega_{0}$, and $\Omega_{c 3} / \omega_{0}$ are found to be $\approx 0.036,0.53$, and 0.63 , respectively.

A direct comparison of the above numbers with those of Ref. [25] is not possible since in that case the annular potential was modeled as a hard-wall potential, while in our study we have assumed harmonic confinement and as a result the density varies in the transverse direction. According to [25], neglecting logarithmic factors of order unity, $\Omega_{c 1} / \omega_{0}$ is $\approx a_{0}^{2} /(2 R d)$, where $d$ is the width of the annulus. Assuming that $d=a_{0}$, then $\Omega_{c 1} / \omega_{0}=1 / 8$, which is roughly a factor of 3.5 larger than the value found numerically. Also, according to the same study $\Omega_{c 2} / \omega_{0}=2\left(a_{0} / d\right)$, which is equal to two, and this differs by a factor of roughly four from the value found above. On the other hand, the ratio $\Omega_{c 1} / \Omega_{c 2}=a_{0} /(4 R)=1 / 16$, which is rather close to the ratio $0.036 / 0.53 \approx 1 / 14.7$.

\section{DIPOLAR CASE}

We turn now to the case where the atoms have a nonzero dipole moment. In addition to the hard-core po-
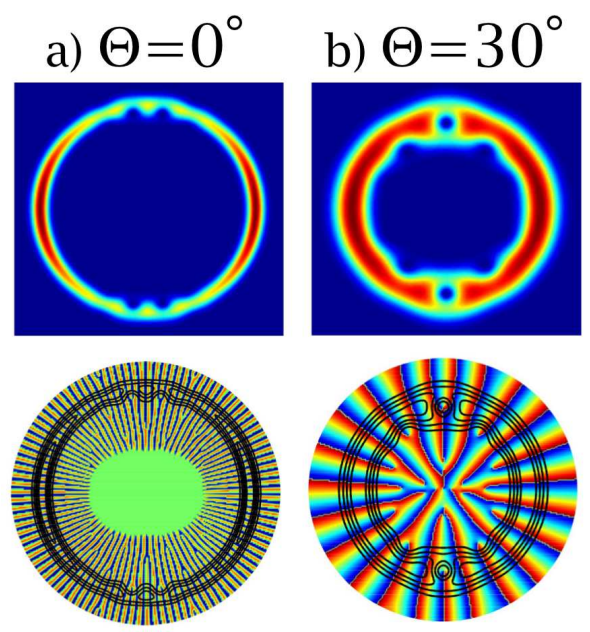

c) $\Theta=60^{\circ}$
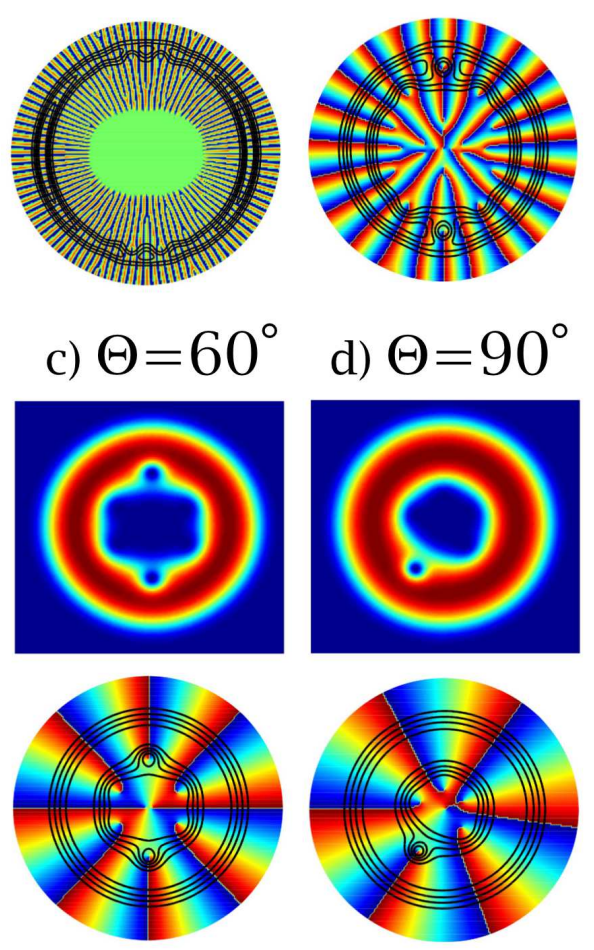

FIG. 3: (Color online) Density and phase of the order parameter corresponding to the critical frequency $\Omega_{c 2}$ for the dipolar case, with $\sqrt{8 \pi} N a / a_{z}=150$, and $a_{d d} / a_{0}=2.505^{2} / 3$. For (a) $\Theta=0^{\circ}, \Omega_{c 2} / \omega_{0} \approx 0.80$, (b) $\Theta=30^{\circ}, \Omega_{c 2} / \omega_{0} \approx 0.5835$, (c) $\Theta=60^{\circ}, \Omega_{c 2} / \omega_{0} \approx 0.36$, and (d) $\Theta=90^{\circ}, \Omega_{c 2} / \omega_{0} \approx 0.296$.

tential considered in the previous section - which is kept fixed, with $M g / \hbar^{2}=\sqrt{8 \pi} N a / a_{z}=150$ - we consider a dipolar interaction of a fixed strength. Introducing the dipolar length $a_{d d} \equiv M D^{2} /\left(3 \hbar^{2}\right)$, we choose $a_{d d} / a=$ $2.505^{2} / 3$. In the above expression $D^{2}=d^{2} /\left(4 \pi \epsilon_{0}\right)$ when the atoms have an electric dipole moment $d$, where $\epsilon_{0}$ is the permittivity of the vacuum; when the atoms have a magnetic moment $\mu, D^{2}=\mu_{0} \mu^{2} /(4 \pi)$, where $\mu_{0}$ is the permeability of the vacuum. With this choice of parameters the dipolar and the contact interactions are of comparable magnitude.

In this problem, small values of $D$ and thus weak dipolar interactions may give rise to a very smooth energy surface, which leads to degeneracy problems in the calculation. On the other hand, large values of $D$ tend to cure this problem, however they also make the system unstable against collapse for small values of the angle $\Theta$ due to the head-to-tail alignment of the dipoles. 
We investigate the rotational response of the cloud considering four values for the angle of the dipole moment, $\Theta=0^{\circ}, 30^{\circ}, 60^{\circ}$, and $90^{\circ}$, as in Fig. 1 . When $\Theta=90^{\circ}$ the interaction respects the axial symmetry of the trapping potential and is also purely repulsive. As a result, the system behaves qualitatively as in the case of a contact potential alone, with a phantom vortex state, a real vortex state, and a vortex lattice forming with increasing $\Omega$.

For any other value of the angle $\Theta$, as $\Omega$ increases, initially the cloud still responds by forming one phantom vortex at the center of the trap, as in the non-dipolar case. On the other hand, for higher rotational frequencies the gas behaves in a qualitatively different way. In this case the dipolar interaction breaks the axial symmetry of the Hamiltonian, introducing a preferred direction.

In Fig. 3 we plot the density and the phase of the order parameter for four values of the angle $\Theta=0^{\circ}, 30^{\circ}, 60^{\circ}$, and $90^{\circ}$, for the value of the angular frequency that corresponds to $\Omega_{c 2}$, i.e., for the value of $\Omega$ for which the first (real) vortex state penetrates the annulus. While for $\Theta=90^{\circ}$ the position where the vortex enters the annulus is arbitrary (as in the non-dipolar case), for all other values of $\Theta$ this is determined by the direction of the polarizing field, which is chosen to be in the direction going from the bottom to the top of the page. For $\Theta=60^{\circ}$ and $30^{\circ}$ there are actually two vortex states which enter the annulus from opposite ends. For $\Theta=0^{\circ}$ instead of two vortices, there are two pairs of vortices, which are symmetrically displaced from the direction of the polarizing field. As one can see from the phase of the order parameter, as $\Theta$ decreases the number of phantom vortices increases and especially for $\Theta=0^{\circ}$ their number is very large. Also, with decreasing $\Theta$ the density at the center of the trap decreases. For $\Theta=0^{\circ}$ the density in this region is extremely small and therefore it appears to be "empty" in Fig. 3, as it can be seen also from the phase plot.

Furthermore, as we have seen numerically, the anisotropic character of the dipolar interaction affects the frequencies $\Omega_{c 1}$ and $\Omega_{c 2}$ in a different way. For $\Theta=0^{\circ}, 30^{\circ}, 60^{\circ}$ and $90^{\circ}, \Omega_{c 1}$ is found to increase linearly with $\Theta$, with $\Omega_{c 1} / \omega_{0}$ being approximately equal to $0.035,0.036,0.037$, and 0.038 , respectively. Also, $\Omega_{c 2} / \omega_{0}$ is found to be $\approx 0.80,0.5835,0.36$, and 0.296 , respectively. In other words, as $\Theta$ decreases, i.e., as the system gets more distorted from axial symmetry, $\Omega_{c 1}$ decreases slightly (and linearly), whereas $\Omega_{c 2}$ increases. It is interesting that for $\Theta=30^{\circ}, \Omega_{c 1}$ is identical to that of the non-dipolar gas. For this value of $\Theta$ the repulsive and attractive parts of the dipolar interaction are comparable and in a sense balance each other.

\section{SUMMARY}

In the present study we have investigated two problems. First, we studied the rotational response of a su-

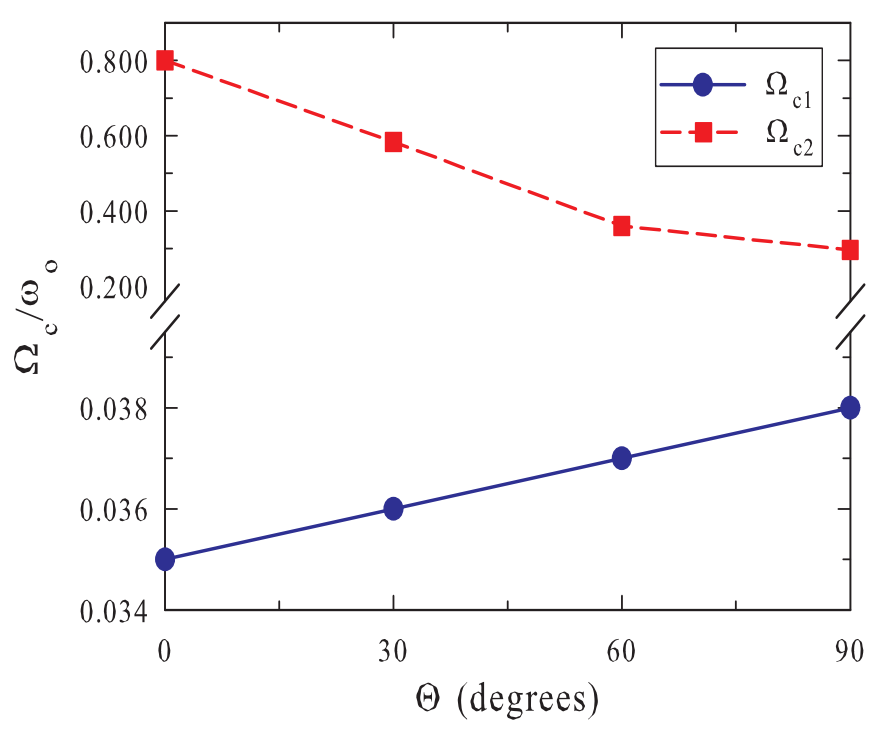

FIG. 4: (Color online) The critical frequencies $\Omega_{c 2}$ and $\Omega_{c 3}$ as a function of the angle $\Theta$ for the parameters of Fig. 3 .

perfluid that is confined in an annular potential. Such a trapping geometry interpolates in a sense between oneand two-dimensional motion, depending on the ratio between the mean radius of the annulus and its width. The second problem we have addressed is the effect of the long-range and anisotropic character of the dipolar interaction.

Starting with the non-dipolar case, as the rotational frequency of the annulus increases, there is a critical frequency above which a vortex state appears in the region of essentially zero density, around the center of the trap. Eventually more vortices start to reside in this region. These vortices affect only the phase of the order parameter, increasing the circulation and leaving the density unaffected. As the rotational frequency increases, the number of "phantom" vortices increases as well. Eventually the number of phantom vortices which can be accommodated in the region of low atom density reaches a threshold. As a result, above some critical frequency, one of these vortices penetrates the annulus, moving to the region of non-zero density. These "real" vortices eventually form a regular vortex array, or even multiple arrays, with increasing $\Omega$.

The dipolar case differs from the above mainly because of the symmetry-breaking of the Hamiltonian due to the dipolar interaction (unless the dipole moment is perpendicular to the plane of motion of the atoms). Even the non-rotating system is affected by the dipole-dipole interaction, with the formation of one or two density maxima, depending on the value of the parameters, as seen in Refs. 12, 14, 15]. This inhomogeneity in the density affects also the critical rotational frequencies. Initially a "phantom" vortex still appears at the center of the trap. However, for larger rotational frequencies of the trap the 
"real" vortices penetrate the annulus either in pairs, or in couples of pairs from opposite ends of the cloud in the direction of the polarizing field of the dipoles.

Rotating atomic Bose-Einstein condensates confined in toroidal potentials have been realized experimentally recently, while dipolar gases are under intense experimental investigation. As shown in the present article, the combination of these two effects - that should be accessible also experimentally - gives rise to interesting effects.

\section{ACKNOWLEDGEMENTS}

We thank Alexander L. Fetter and Kimmo Kärkkäinen for very useful discussions. This work was financed by the Swedish Research Council and originated from a collaboration within the POLATOM Research Networking Programme of the European Science Foundation (ESF). E. Ö. K. is supported by the Turkish Council of Higher Education (YÖK) within the scope of Post-Doctoral Research Scholarship Program.
[1] S. Gupta, K. W. Murch, K. L. Moore, T. P. Purdy, and D. M. Stamper-Kurn, Phys. Rev. Lett. 95, 143201 (2005); S. E. Olson, M. L. Terraciano, M. Bashkansky, and F. K. Fatemi, Phys. Rev. A 76, 061404(R) (2007); K. Henderson, C. Ryu, C. MacCormick, and M. G. Boshier, New J. Phys. 11, 043030 (2009).

[2] C. Ryu, M. F. Andersen, P. Cladé, V. Natarajan, K. Helmerson, and W. D. Phillips, Phys. Rev. Lett. 99, 260401 (2007).

[3] A. Ramanathan, K. C. Wright, S. R. Muniz, M. Zelan, W. T. Hill, C. J. Lobb, K. Helmerson, W. D. Phillips, and G. K. Campbell, Phys. Rev. Lett. 106, 130401 (2011).

[4] M. Gildemeister, B. E. Sherlock, and C. J. Foot Phys. Rev. A 85, 053401 (2012).

[5] Stuart Moulder, Scott Beattie, Robert P. Smith, Naaman Tammuz, and Zoran Hadzibabic, eprint condmat/1112.0334.

[6] S. Viefers, P. Koskinen, P. Singha Deo, and M. Manninen, Physica E (Amsterdam) 21, 1 (2004).

[7] Manninen et al., to be published.

[8] A. Griesmaier, J. Werner, S. Hensler, J. Stuhler, and T. Pfau, Phys. Rev. Lett. 94, 160401 (2005); J. Stuhler, A. Griesmaier, T. Koch, M. Fattori, T. Pfau, S. Giovanazzi, P. Pedri, and L. Santos, ibid. 95, 150406 (2005); M. Fattori, T. Koch, S. Goetz, A. Griesmaier, S. Hensler, J. Stuhler, and T. Pfau, Nature Phys. 2, 765 (2006); A. Griesmaier, J. Stuhler, T. Koch, M. Fattori, T. Pfau, and S. Giovanazzi, Phys. Rev. Lett. 97, 250402 (2006).

[9] K. -K. Ni, et al., Science 322, 231 (2008); S. Ospelkaus, K. -K. Ni, G. Quemener, B. Neyenhuis, D. Wang, M. H. G. deMiranda, J. L. Bohn, J. Ye, and D. S. Jin, Phys. Rev. Lett. 104, 030402 (2010).

[10] T. Lahaye, C. Menotti, L. Santos, M. Lewenstein, and T. Pfau, Rep. Prog. Phys. 72, 126401 (2009).

[11] G. M. Brunn and E. Taylor, Phys. Rev. Lett. 101, 245301 (2008).
[12] M. Abad, M. Guilleumas, R. Mayol, M. Pi, and D. M. Jezek, Phys. Rev. A 79, 063622 (2009).

[13] F. Malet, T. Kristensen, S. M. Reimann, and G. M. Kavoulakis, Phys. Rev. A 83, 033628 (2011).

[14] M. Abad, M. Guilleumas, R. Mayol, M. Pi, and D. M. Jezek, Phys. Rev. A 81, 043619 (2010).

[15] F. Malet, G. M. Kavoulakis, and S. M. Reimann, Phys. Rev. A 84, 043626 (2011).

[16] M. Abad, M. Guilleumas, R. Mayol, M. Pi, and D. M. Jezek, Phys. Rev. A 84, 035601 (2011).

[17] M. Abad, M. Guilleaumas, R. Mayol, M. Pi, and D. M. Jezek, Europhys. Lett. 94, 10004 (2011).

[18] M. Abad, M. Guilleaumas, R. Mayol, M. Pi, and D. M. Jezek, Laser Physics 18, 648 (2008).

[19] Mingwu Lu, Nathaniel Q. Burdick, Seo Ho Youn, and Benjamin L. Lev, Phys. Rev. Lett. 107, 190401 (2011).

[20] Sascha Zöllner, G. M. Bruun, C. J. Pethick, and S. M. Reimann, Phys. Rev. Lett. 107, 035301 (2011).

[21] B. Xiong, J. Gong, H. Pu, W. Bao, and B. Li, Phys. Rev. A 79, 013626 (2009); M. Asad-uz-Zaman and D. Blume, Phys. Rev. A 80, 053622 (2009).

[22] F. Piazza, L. A. Collins, and A. Smerzi, Phys. Rev. A 80, 021601 (2009).

[23] R. Dubessy, T. Liennard, P. Pedri, and H. Perrin, Phys. Rev. A 86, 011602 (2012).

[24] S. J. Woo and Young-Woo Son, Phys. Rev. A 86, 011604(R) (2012).

[25] Alexander L. Fetter, Phys. Rev. 153, 285 (1967).

[26] P. J. Bendt and R. J. Donnelly, Phys. Rev. Lett. 19, 214 (1967).

[27] J. C. Cremon, G. M. Bruun, and S. M. Reimann, Phys. Rev. Lett. 105, 255301 (2010).

[28] S. A. Chin and E. Krotscheck, Phys. Rev. E 72, 036705 (2005). 